\title{
Epidemiologia del suicidio in Italia. Problemi metodologici ed analisi dei dati aggregati
}

\author{
PAOLO CREPET
}

Solo qualche anno fa sarebbe sembrato inusuale leggere in una rivista psichiatrica un editoriale sull'epidemiologia delle condotte suicidarie; ma oggi non è più così. Negli ultimi anni, infatti, il continuo peggioramento dei dati statistici riguardanti le morti per suicidio non solo ha costituito un doveroso - e non sempre ascoltato - richiamo per le autorità deputate alla salvaguardia della salute pubblica, ma ha anche rappresentato un rinnovato stimolo per ricercatori e clinici. La rinascita di tale interesse è sicuramente legata anche al nuovo assetto organizzativo che la psichiatria italiana si è data con l'approvazione della riforma del 1978. Il superamento dell'isolamento del campo psichiatrico dal resto dell'organizzazione dei servizi sanitari e sociali - avvenuto attraverso il rafforzamento dei rapporti con la medicina di base ed ospedaliera e la diffusione dei servizi territoriali di salute mentale - ha permesso, infatti, un enorme ampliamento del suo osservatorio nella comunità sociale fino a identificare nuovi fattori di rischio psicopatologico, come quelli correlati alle condotte autodistruttive.

Nonostante l'accresciuto interesse scientifico, lo studio delle condotte suicidarie in Italia è reso complesso dalla difficoltà a reperire dati statistici affidabili. La divisione delle statistiche elaborate dall'ISTAT rispetto alla fonte (giudiziaria e sanitaria) non agevola certo il lavoro dei ricercatori, ma ciò che lo aggrava è la scarsezza dei dati raccolti routinariamente e l'impossibilità di incrociarli con variabili individuali, sociali e cliniche. Ne emerge un quadro interlocutorio che rimanda necessariamente alle ricerche locali ogni successivo approfondimento.

Indirizzo per la corrispondenza: Dr. P. Crepet, Via della Gensola, 38, 00153 Roma.

Fax (+39) 06-583.67.36.
Tuttavia anche sull'interpretazione dei dati aggregati a livello nazionale emergono differenze rilevanti. L'ISTAT, attraverso le fonti giudiziarie, non fornisce tassi di suicidio ma solo dati grezzi: ciò è all'origine della pubblicazione di dati significativamente diversi tra loro (soprattutto riguardo la disaggregazione per classi d'età riferita agli anni più recenti). La confusione aumenta per quanto riguarda i tassi standardizzati per età (calcolo assolutamente indispensabile per l'esame dei trend temporali in quanto il suicidio è fortemente correlato con l'età): spesso vi è disomogeneità nella modalità di standardizzazione (diretta e indiretta), non si utilizzano le stesse classi d'età, né si fa riferimento alla stessa fonte (quella sanitaria - che fornisce un dato più attendibile in quanto prende in esame anche le morti per suicidio avvenute a distanza di tempo dall'evento - pubblica con molto ritardo rispetto a quella giudiziaria e per ciò spesso non viene scelta).

Accanto ai problemi metodologici vi sono anche quelli legati alla classificazione e, dunque, alla stima dell'entità reale del fenomeno. La sua grossolana sottostima è legata ad almeno quattro categorie «sospette»: le morti per overdose (l'OMS stima che almeno un terzo siano veri e propri suicidi), le morti per incidente stradale (soprattutto quando la vettura ha il solo conducente a bordo), quelle accidentali (in particolare quelle domestiche) e quelle causate da malattie mentali. I tassi di queste ultime sono in costante ascesa, ma ciò è solo in parte spiegabile con l'invecchiamento della popolazione (tra queste morti sono incluse quelle provocate da psicopatologia senile su base organica) in quanto tale incremento è ancor più evidente tra le persone con meno di $\mathbf{3 0}$ anni tra $i$ quali i tassi di mortalità per malattia mentale hanno, dal 1987, superato quelli per suicidio (Crepet, in stampa). 
Ciò premesso, l'analisi dei dati aggregati dimostra che in Italia il trend in questo secolo non si discosta di molto da quello registrato in altri paesi europei (Bille-Brahe \& Crepet, in stampa); almeno due sono, infatti, i punti in comune: l'incremento registrato tra la fine degli anni ' 20 e l'inizio degli anni ' 30 (durante il quale $i$ tassi hanno raggiunto $i$ valori più alti in assoluto) e il calo in corrispondenza dei due eventi bellici (Crepet \& Florenzano, 1989; Crepet et al., 1991).

I dati relativi al periodo 1959-1989 dimostrano che la crescita dei tassi di suicidio standardizzati per età $(10,7 \%)$ è quasi esclusivamente attribuibile alla popolazione maschile $(17,8 \%$, il tasso nel 1989 è di 13,9 per 100.000), mentre quella registrata tra le femmine è stata assai più modesta $(6,2 \%$, il tasso è di 5,1$)$ (Crepet, in stampa); in contrasto con la letteratura internazionale (Platt, 1988), dunque, in Italia non sembra verificarsi la tendenza ad un riequilibrio del rapporto maschi/femmine. Inoltre, alcune indagini escludono che la crescita dei valori registrata negli anni ' 80 sia attribuibile alla riforma psichiatrica (Williams et al., 1986; Tansella, 1988).

Per quanto riguarda l'età, mentre tra le classi d'età più giovani i tassi non dimostrano incrementi significativi, tra gli anziani il quadro è drammatico: tra il 1970 e il 1988 tra gli ultrasettantenni il valore dei tassi è cresciuto del $43,5 \%$.

Tuttavia, per una migliore comprensione del fenomeno suicidario in Italia occorre disaggregare i tassi standardizzati per età per aree geografiche. Emerge, infatti, che nel nord-est $i$ valori sono tre volte più alti rispetto al sud; questo dato è confermato anche dall'analisi dei valori riferiti alle singole regioni, con le sole eccezioni della Basilicata e della Sardegna dove $i$ valori sono significativamente più alti di quelli registrati nelle regioni vicine (Biggeri et al., 1990; Crepet et al., 1991). Tali differenze possono essere attribuite - oltre ai fattori culturali, economici e sociali - anche all'emigrazione: oltre il $20 \%$ dei residenti nelle regioni meridionali commettono il suicidio in quelle dove sono emigrati; nell'esame delle differenze geografiche è quindi importante verificare se il dato si riferisce al luogo dove è avvenuta la morte o a quello della residenza anagrafica del suicida (Crepet \& Florenzano, 1990).

Un altro aspetto interessante anche per il suo risvolto clinico riguarda l'andamento della stagionalità dei tassi di suicidio (molti autori correlano tali dati con quelli riferiti alla presenza di alcuni mediatori cerebrali legati alla patologia affettiva). Anche in Italia è stato confermato l'andamento ciclico dell'incidenza del suicidio riscontrato a livello internazionale (con apogeo a maggio ed ipogeo a novembre); tale andamento non dimostra diferenze rispetto alle aree geografiche, ma rispetto all'ambiente urbano del centro/nord e quello rurale del sud. Il che significa che le differenze climatiche non influiscono sull'incidenza stagionale del suicidio, al contrario di quanto accade per i fattori ambientali, più diffusi nelle aree urbane (Micciolo et al., 1988; Micciolo et al., 1989; Micciolo et al., 1991).

Un'ulteriore interpretazione dell'andamento del fenomeno suicidario può essere fornita dagli studi sulla correlazione con i tassi di disoccupazione. Tale rapporto è stato, infatti, utilizzato per interpretare sia il fenomeno emerso in corrispondenza della grande recessione economica del 1929 (Smith, 1987) sia la crisi economica dell'ultimo ventennio (Platt, 1988). In Italia, tuttavia, i risultati di analoghe ricerche sono stati alquanto contraddittori. Da un lato, l'unico studio longitudinale di mortalità finora eseguito ha messo in evidenza che lo stato di disoccupazione rappresenta un importante fattore di rischio di morte, in particolare per suicidio: a parità di sesso, età e condizione sociale chi ha perso il lavoro ha una probabilità 3 volte superiore di suicidarsi rispetto all'occupato (Costa et al., 1989). Dall'altro lato, mentre alcune ricerche hanno messo in luce una correlazione positiva tra i tassi di suicidio e di disoccupazione tra i maschi e negativa tra le femmine (tra le quali pesa però «l'inquinamento» dei dati creato dall'incerta classificazione delle casalinghe) (Crepet \& Florenzano, 1987; Florenzano \& Crepet, 1988), altre indagini inducono a conclusioni più caute (Micciolo, 1990; Platt et al., in stampa, a; b). Un aspetto interessante che è emerso da queste ricerche riguarda l'esistenza di una correlazione negativa tra tassi di suicidio e tassi di disoccupazione nella distribuzione geografica: $i$ tassi di suicidio sono più alti là dove quelli di disoccupazione sono più bassi. Una possibile interpretazione di tale fenomeno riguarda il minor stigma sociale avvertito da chi vive in aree dove la disoccuapzione è un fenomeno «endemico» (Platt \& Kreitman, 1984).

Maggiori certezze sull'interpretazione delle correlazioni possibili tra il fenomeno suicidario e gli indicatori socialmente più rilevanti - e tra questi certamente la disoccupazione - potranno comunque pervenire dall'ulteriore sviluppo di indagini individuali trasversali e longitudinali. 


\section{BIBLIOGRAFIA}

Biggeri A., Ferrara M. \& Stefanini P. (1990). Epidemiologia descrittiva del suicidio in Italia. In La Salute degli Italiani. Rapporto 1990 (ed. M. Geddes), pp. 81-94. La Nuova Italia Scientifica: Roma.

Bille-Brahe U. \& Crepet P. Trend in the frequency of suicide in Denmark and in Italy. In Suicidal Behavior in Europe. Recent Research Trends (ed. P. Crepet, G. Ferrari, S. Platt and M. Bellini). John Libbey: London (in stampa).

Costa G., Crepet P. \& Florenzano F. (1989). Unemployment and mortality in Italy. The Turin longitudinal study. In Current Research on Suicide and Parasuicide (ed. N. Kreitman and S. Platt), pp. 40-46. Edinburgh University Press: Edinburgh. Crepet $P$. Suicide trends in Italy. New epidemiological findings. European Psychiatry (in stampa).

Crepet P. \& Florenzano F. (1989). Il Rifiuto di Vivere. Anatomia del Suicidio. Editori Riuniti: Roma.

Crepet P. \& Florenzano F. (1990). Epidemiology of suicide in Italy: an update. In Suicidal Behaviour and Risk Factors (ed. G. Ferrari, M. Bellini and P. Crepet), pp. 65-70. Monduzzi: Bologna.

Crepet P., Caracciolo S., Casoli R., Fabbri D., Florenzano F., Grassi G. M., Jonus A. \& Tomelli A. (1991). Suicidal behavior in Italy: data, trends and guidelines for a suicide intervention/prevention policy. Suicide and Life-Threatening Behavior $21,263-278$.

Florenzano F. \& Crepet P. (1987). Suicidio e disoccupazione. Analisi del rischio attribuibile e delle correlazioni. Rivista Sperimentale di Freniatria 111, 7-20.

Micciolo R. (1990). Suicidio e disoccupazione in Italia negli anni
1979-1988. Quaderni di Statistica e Matematica Applicata alle Scienze Economico-Sociali 3, 75-96.

Micciolo R., Zimmermann-Tansella Ch., Williams P. \& Tansella M. (1988). Geographical variation in the seasonality of suicide. Journal of Affective Disorders 15, 163-168.

Micciolo R., Zimmermann-Tansella Ch., Williams P. \& Tansella M. (1989). Seasonal variation in suicide: is there a sex difference? Psychological Medicine 19, 199-203.

Micciolo R., Zimmermann-Tansella Ch., Williams P. \& Tansella M. (1991). Geographical and urban-rural variation in the seasonality of suicide: some further evidence. Journal of Affective Disorders 21, 39-43.

Platt S. (1988). Suicide trends in 24 European countries, 1972-1984. In Current Issues of Suicidology (ed. H. J. Moller, A. Schmidtke and R. Welz), pp. 3-13. Springer: Berlin.

Platt S. \& Kreitman N. (1984). Trends in parasuicide and unemployment among men in Edinburgh, 1968-82. British Medical Journal 289, 1029-1032.

Platt S., Micciolo R. \& Tansella M. Suicide and unemployment in Italy: description, analysis and interpretation of recent trends. Social Science and Medicine (in stampa, a).

Platt S., Micciolo R. \& Tansella M. Suicide and unemployment in Italy: a replication study. In Suicidal Behavior in Europe. Recent Research Trends (ed. P. Crepet, G. Ferrari, S. Platt and $M$. Bellini). John Libbely: London (in stampa, b).

Smith R. (1987). Unemployment and Health. A Disaster and a Challenge. Oxford University Press: Oxford.

Tansella M. (1988). L'epidemiologia del suicidio in Italia. Analisi dei dati nazionali e regionali. In Il Suicidio nel Mondo Contemporaneo. Aspetti Bio-Psico-Sociali (ed. L. Pavan e D. De Leo), pp. 3-15. Liviana: Padova.

Williams P., De Salvia D. \& Tansella M. (1986). Suicide, psychiatric reform and the provision of psychiatric services in Italy. Social Psychiatry 21, 89-95. 


\section{PeriodicaMente}

